\title{
Response rate and safety in patients with hepatocellular carcinoma treated with transarterial chemoembolization using $40-\mu \mathrm{m}$ doxorubicin-eluting microspheres
}

\author{
Katharina Carolin Albrecht ${ }^{1} \cdot$ René Aschenbach $^{1} \cdot$ Ioannis Diamantis ${ }^{1} \cdot$ Niklas Eckardt $^{1} \cdot$ Ulf Teichgräber $^{1}$ (I)
}

Received: 2 March 2020 / Accepted: 19 August 2020 / Published online: 2 September 2020

(c) The Author(s) 2020

\begin{abstract}
Purpose To evaluate the response rate and safety of superselective drug-eluting beats transarterial chemoembolization (DEBTACE) with doxorubicin-loaded 40- $\mu \mathrm{m}$ microspheres in patients with hepatocellular carcinoma (HCC).

Methods One hundred and forty-one treatments with doxorubicin-loaded 40- $\mu \mathrm{m}$ microspheres in 83 patients between 2012 and 2017 were retrospectively evaluated. Images of the treated lesions were analyzed before and after each treatment according to mRECIST (modified Response Evaluation Criteria in Solid Tumors). Therapy response (complete response $[\mathrm{CR}]+$ partial response $[\mathrm{PR}])$ and disease control (CR + PR + stable disease [SD]) rates were determined, and the correlation between the longitudinal axis (longest diameter of the tumor) and volume was investigated using a newly developed software for systematic tumor response assessment. Additional endpoints were progression-free survival (PFS) and time to progression (TTP).

Results In the target tumors, a therapy response rate of $63.1 \%$ and a disease control rate of $95.7 \%$ were achieved. There was a good correlation between the measurement of the longitudinal axis and volume of the measured lesion ( $r$ value, 0.954). The median PFS was 2.23 months, and the median TTP was 5.91 months. The serious adverse event rate (SAE) was 10.64\%. Conclusion Superselective DEB-TACE with $40-\mu \mathrm{m}$ sized Embozene Tandem ${ }^{\mathrm{TM}}$ can be considered an effective and safe treatment, given the number of procedure-related complications.
\end{abstract}

Keywords Transarterial chemoembolization $\cdot$ TACE $\cdot$ Hepatocellular carcinoma $\cdot$ Doxorubicin-eluting microspheres

Electronic supplementary material The online version of this article (https://doi.org/10.1007/s00432-020-03370-z) contains supplementary material, which is available to authorized users.

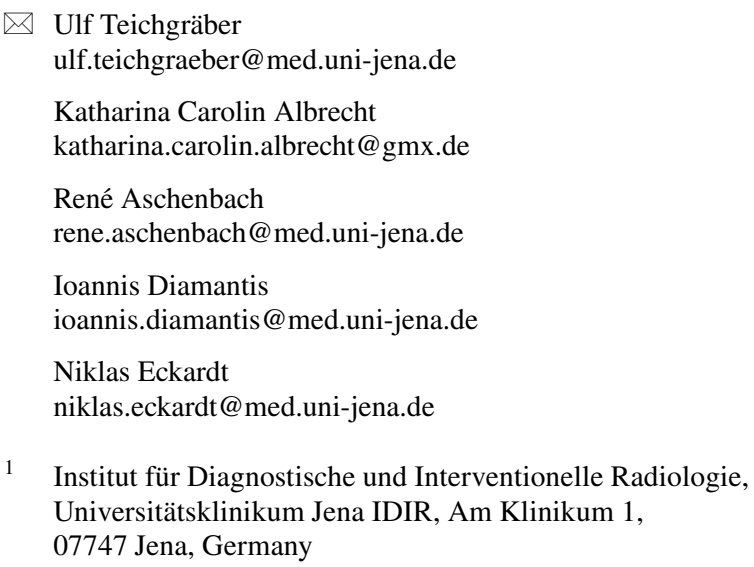

$\begin{array}{ll}\text { Abbreviations } \\ \text { DEB } & \text { Drug-eluting beats } \\ \text { TACE } & \text { Transarterial chemoembolization } \\ \text { HCC } & \text { Hepatocellular carcinoma } \\ \text { CR } & \text { Complete response } \\ \text { PR } & \text { Partial response } \\ \text { SD } & \text { Stable disease } \\ \text { PFS } & \text { Progression-free survival } \\ \text { TTP } & \text { Time to progression } \\ \text { SAE } & \text { Serious adverse event } \\ \text { MRI } & \text { Magnetic resonance imaging } \\ \text { CT } & \text { Computed tomography } \\ \text { AFP } & \text { Alpha-fetoprotein } \\ \text { PD } & \text { Progressive disease } \\ \text { MPP } & \text { Mean value of positive pixels } \\ \text { UPP } & \text { Uniformity of distribution of positive pixels } \\ \text { mRECIST } & \text { Modified response evaluation criteria in solid } \\ & \text { tumors } \\ \text { AE } & \text { Adverse events }\end{array}$


PES Post-embolization syndrome

c-TACE Conventional TACE

\section{Introduction}

The current standard treatment for early and intermediatestage hepatocellular carcinoma (HCC) is transarterial chemoembolization (TACE) (Lammer et al. 2010). Nearly half of all patients with $\mathrm{HCC}$ receive TACE at some time point during the course of their disease (Lencioni et al. 2014). TACE is a multifaceted treatment including several different technical approaches. Conventional TACE was the first established method. The technique uses lipiodol mixed with antineoplastic emulsion (Han et al. 2014). Two randomized controlled trials have illustrated the survival benefits of conventional TACE over supportive care (Dhanasekaran et al. 2010). Later, a new concept of drug-eluting embolic transarterial chemoembolization (DEB-TACE) using microspheres was introduced (Huppert 2011). This technique effectively combines enhanced local drug delivery and ischemic embolization effects (Huppert 2011). Therefore, the chemotherapeutic agent is tightly connected to the microspheres. The microspheres have been developed for transcatheter treatment of HCC to deliver higher doses of the chemotherapeutic agent and to prolong contact time with the tumor (Nam et al. 2016). First, they were available in sizes ranging between 500 and $300 \mu \mathrm{m}$ to deliver a high amount of chemotherapeutic agent into the target tumor. In time, smaller sized microspheres were developed that can reach into smaller tumor feeding vessels with a potentially superior efficacy and increased necrosis of the target tissue (Sattler et al. 2018). Throughout the years, the development led to sizes ranging between 100 and $70 \mu \mathrm{m}$ and finally the extremely small 40- $\mu \mathrm{m}$ microspheres. The size distribution allows for better steerable vessel occlusion that minimizes the undesirable side effects of a non-target embolization.

This retrospective study examined procedure-related complications in relation to the therapeutic response by evaluating pre-and post-treatment magnetic resonance imaging (MRI) and computed tomography (CT) scans after DEBTACE with $40 \mu \mathrm{m}$-sized microspheres. In addition, radiological image histograms, classification, and further clinical parameters were evaluated.

\section{Materials and methods}

\section{Study design}

This retrospective study was conducted in a tertiary care university hospital and was approved by the local institutional review board. The requirement for informed consent was waived due to the retrospective nature of the study. The observation period was from May 2012 to May 2017.

\section{Patients}

The study population consisted of 137 consecutive adult patients with HCC who received DEB-TACE with $40 \mu \mathrm{m}$-sized microspheres. The exclusion and inclusion criteria are shown in Table 1. Eighty-three patients were enrolled. The characteristics of the patients are summarized in Table 2 (women, 8 ; men, 75; mean age, 64.7 years; range 32-84 years). Forty-eight patients received an HCC diagnosis confirmed by histological examination. Thirty-five patients were diagnosed with $\mathrm{HCC}$ using at least 2 imaging modalities (CT, MRI, or ultrasonography) $(n=24)$ or by use of 1 liver imaging modality combined with an increased level of alpha-fetoprotein (AFP) $(>20 \mathrm{ng} / \mathrm{ml})(n=11)$ (Abbasi et al. 2012).

The indications for DEB-TACE treatment were bridging for transplantation in $49(59 \%)$ cases, palliative treatment in $29(35 \%)$ cases, and as a curative approach in $5(6 \%)$ cases, as the size of the index tumor was not major at the time of diagnosis. Forty-two patients fulfilled the Milan criteria (a single tumor $5 \mathrm{~cm}$ or less in size or up to 3 tumors, each $3 \mathrm{~cm}$ or less in size, and no macroscopic vascular invasion

Table 1 Inclusion and exclusion criteria

\begin{tabular}{ll}
\hline Inclusion criteria & Exclusion criteria \\
\hline Only the usage of $40 \mu \mathrm{m}$ sized microspheres during DEB-TACE & Different particle size $(100$ or $75 \mu \mathrm{m})$ \\
Liver imaging before and after each treatment & Lipiodol application (conventional TACE) \\
No other simultaneous treatment & Missing follow-up investigation \\
- Therapy with sorafenib (Nexavar $\left.{ }^{\mathrm{TM}}\right)$ & Different therapy directly after TACE \\
- Radioembolization & Liver transplantation shortly after TACE (median: 30.5 days) \\
- Surgical resection & Therapy with sorafenib (nexavar) \\
- Conventional TACE with lipiodol (c-TACE) & Missing arterial phase imaging in follow up investigation \\
- Other particle sizes & Extensive target lesion \\
\hline
\end{tabular}

$D E B-T A C E$ drug-eluting embolic transarterial chemoembolization, $c-T A C E$ conventional TACE 
Table 2 Demographic characteristics and tumoral parameters of the study population $(n=83)$

\begin{tabular}{ll}
\hline Age & 64.7 years $(32-84$ years $)$ \\
Sex & 75 male, 8 female
\end{tabular}

Child-Pugh Score

A

ECOG Score

BCLC stages

Tumor extension

Tumor size

$36.78 \pm 28.20 \mathrm{~mm}$

(minimum: $7.50 \mathrm{~mm}$, maximum 172.10)

Number of tumor lesions

138

Number of lesions per patient

$1.35 \pm 0.688$ (minimum: 1 , maximum 4)

Unilobar

$n$

Bilobar

22

DEB-TACE re-treatment cycle

Alcohol intake

Amount of patients $(n=90)^{1}$

Hepatitis

-HBV B

-HBV C

Hemochromatosis

Autoimmune hepatitis

9

Nonalcoholic steatohepatitis

2

Unknown origin

Non cirrhotic liver

4

ECOG Eastern Co-operative Oncology Group, BCLC Barcelona Clinic Liver Cancer, DEB-TACE drugeluting embolic transarterial chemoembolization, $H B V$ hepatitis $\mathrm{B}$ virus, $H B C$ hepatitis $\mathrm{C}$ virus

(Mazzaferro et al. 2009)); 41 patients did not fulfill the criteria. The Barcelona Clinic Liver Cancer (BCLC) staging, which connects the disease stage, liver function, and health status to a certain treatment was also applied as shown in Table 2. Further information regarding the etiology of the underlining liver disease is also shown in Table 2. A total of 30 patients had received previous treatment before inclusion with a mean of $7.7 \pm 8.0$ months (range 1.1-37.9 months) having elapsed between the prior treatment and the DEBTACE (Online Resource 1). 


\section{DEB-TACE procedure}

The DEB-TACE was performed by four interventional radiologists with more than 5 years of experience (mean, 16 years; range 11-25 years). The embolization was performed with a coaxial superselective, subsegmental technique using a $5 \mathrm{~F}$ cobra or $5 \mathrm{~F}$ sidewinder catheter, followed by the positioning of a microcatheter (Progreat 2.8, Tokyo, Japan), which was placed as close as possible to the arterial vessel supplying the tumor. One milliliter of Tandem Embozene $^{\mathrm{TM}}$ (Boston Scientific, Marlborough, MA, USA) $40-\mu \mathrm{m}$ microspheres was loaded with $50 \mathrm{mg}$ doxorubicin and diluted in 4-ml ionic contrast medium (Solutrast ${ }^{\mathrm{TM}} 250 \mathrm{mg}$, Bracco, Milan, Italy). The radiologist used either 2-ml Tandem Embozene ${ }^{\mathrm{TM}}$ (with $100 \mathrm{mg}$ doxorubicin) in a 10-ml syringe or 3-ml Tandem Embozene ${ }^{\mathrm{TM}}$ (with $150 \mathrm{mg}$ doxorubicin) in a 15-ml syringe. For large tumors $>3 \mathrm{~cm}$, or more than three nodules adding to a diameter $>3 \mathrm{~cm}, 3-\mathrm{ml}$ vials of microspheres were used. The mixture was slowly injected using 1-ml syringes until blood flow stasis was achieved in the target vessel.

\section{Image evaluation}

Image evaluation was performed using the software Mint Lesion $^{\mathrm{TM}}$ for therapeutic response evaluation (Mint Medical, Dossenheim, Germany). The software allows for efficient workflow and supports radiologists in reporting the imaging studies. It applies the mRECIST criteria precisely by differentiating between complete response (CR), partial response (PR), stable disease (SD), and progressive disease (PD). CR is the disappearance of any intratumoral arterial enhancement in all target lesions.

PR is at least a $30 \%$ decrease in the sum of diameters of viable (contrast enhancement in the arterial phase) target lesions, taking reference the baseline sum of the diameters of target lesions.

PD is an increase of at least $20 \%$ in the sum of the diameters of viable (enhancing) target lesions, taking as reference the smallest sum of the diameters of viable (enhancing) target lesions recorded since the treatment started.

SD defines any cases that do not qualify for either PR or PD (Lencioni and Llovet 2010).

Therefore, it was essential to upload the pre-and postinterventional images and to detect the HCC lesions. In addition, the viable fraction of the HCCs with arterial enhancement was circumnavigated manually in each axial layer, and the size of the circumscribed area was calculated by adding the separate surfaces through Mint Lesion ${ }^{\mathrm{TM}}$ automatically. After this classification, Mint Lesion ${ }^{\mathrm{TM}}$ calculated the percentile alteration for all parameters in the histogram analysis (mean, entropy, kurtosis, mean value of positive pixels (MPP), skewness, standard deviation, uniformity of distribution of positive pixels (UPP), and uniformity), leading to the calculation of the tumor response (CR, PR, SD, PD) according to the mRECIST criteria (Online Resource 2).

The period between the CT or MRI scans before treatment and DEB-TACE was $23.0 \pm 18.2$ days. One hundred and forty-one TACE interventions were performed in 83 patients. This led to a total of 282 follow up examinations, which consisted of $255 \mathrm{CT}$ scans and 27 MRI scans. The interval between re-treatments was in months: $4.4 \pm 36.7$. Criteria for re-treatment was the result of SD, PR or PD in the follow-up scans.

Despite applying the bolus tracking technique in CT imaging, the arterial phase did not always allow for precise detection of the hyperdense HCCs. The mRECIST criteria were obtained by measuring peak enhancement for the nonarterial cohort. It was necessary to use the portal venous phase in $13 \mathrm{CT}(5.1 \%)$ scans $(n=13 / 255)$ and the venous phase in $6 \mathrm{CT}(2.3 \%)$ scans $(n=6 / 255)$. In most cases, the HCCs were evaluated in the arterial phase $(n=236 / 255)$ $(92.6 \%)$.

The analysis in Mint Lesion ${ }^{\mathrm{TM}}$ was based on two different mRECIST evaluations. First, as DEB-TACE is a locoregional procedure, a target-based evaluation of only treated lesions (classified as "target lesions") was performed (target response, Table 3). Untreated lesions were declared to
Table 3 Results for both analysis

\begin{tabular}{llllll}
\hline & Target response & & $\begin{array}{l}\text { Overall } \\
\text { response }\end{array}$ & & $\begin{array}{l}\text { Comparison of target } \\
\text { response and overall } \\
\text { response }\end{array}$ \\
\hline CR & $n$ & $\%$ & $n$ & $\%$ & \\
PR & 56 & 39.72 & 47 & 33.33 & - \\
SD & 33 & 23.40 & 37 & 26.24 & + \\
PD & 46 & 32.62 & 50 & 35.46 & + \\
RR $(C R+P R)$ & 6 & 4.26 & 7 & 4.97 & + \\
DCR $(C R+P R+S D)$ & 135 & 63.12 & 84 & 59.57 & \\
\hline
\end{tabular}

$S D$ stable disease, $P D$ progressive disease, $C R$ complete response, $P R$ partial response, $R R$ response rate, $D C R$ disease control rate 
be "findings". A second analysis was performed respecting all HCCs, regardless of the status of treatment (overall response, Table 3).

\section{Safety profile}

All adverse events (AE) were graded for all 141 DEBTACE interventions and the use of the investigational product according to the Common Terminology Criteria for Adverse Events v 5.0. The safety evaluation was performed by examining if an $\mathrm{AE}$ was procedure-related or product related. A serious AE (SAE) based on the FDA's regulatory definition denotes an $\mathrm{AE}$ that results in a health outcome of death, disability, or hospitalization is life-threatening, or requires intervention to prevent harm (Moore et al. 2007). Death within 30 days after the DEB-TACE intervention was regarded as an SAE. Hospitalization for longer than 3 days was considered as prolongation.

Post-embolization syndrome (PES) was defined as a DEB-TACE-related side effect. PES includes liver enzyme abnormalities, fever, abdominal pain, vomiting, and nausea (Lencioni et al. 2016).

\section{Statistical analysis}

All calculations were performed with the statistical software SPSS $^{\mathrm{TM}}$ (Version 24, IBM, Armonk, NY, USA). The data are presented as median and standard deviation. The compiled parameters from the histogram analysis and laboratory data, which were collected before and after each treatment cycle, were analyzed using the Wilcoxon test. The correlation between the longitudinal axis and volume was evaluated using Spearman's test. The outcome parameters were overall survival (OS), progression-free survival (PFS), and time to progression (TTP). All parameters were calculated with the Kaplan-Meier method. The overall survival (OS) was calculated from the date of the first DEB-TACE procedure to death/latest follow-up. PFS was defined as the period from the first treatment until objective tumor progression (an increase of at least $\geq 20 \%$ in the viable target lesion) or death (Nakamura et al. 2012). TTP was defined as the period from the first treatment until objective tumor progression (an increase of at least $\geq 20 \%$ in the viable target lesion) not considering the event "death" (Saad 2008). Both PFS and TTP were determined after each treatment cycle.

\section{Results}

\section{DEB-TACE}

The mean number of treatments per patient was 1.7. The average amount of doxorubicin in each intervention was $75.00 \pm 53.25 \mathrm{mg}$ (minimum, $2.2 \mathrm{mg}$; maximum, $150.00 \mathrm{mg})$.

\section{Survival}

A total of 28 patients (33.7\%) died after $\mathrm{HCC}$ was diagnosed (range, 1.7-57.3 months). In this group, 13 patients with CR (46.43\%), 7 with SD (25.00\%), 6 with PR (21.43\%), and 2 with PD (7.14\%) died. Overall six patients were diagnosed with PD. Despite the fact, that 49 patients were submitted to TACE for bridging, only 14 patients received a liver transplant (reasons for drop-out are listed in Online Resource 3 ). The period from the listing to receiving the organ was $9.2 \pm 3.9$ months. Despite receiving a liver transplant, four patients died. One patient that received a liver transplant had an extra hepatic relapse (pulmonary and lymphogenous metastasis) 2 years later. Therapy with sorafenib was started but was not successful. Among the 69 patients who did not receive a liver transplant, the OS (Fig. 1) was $83.2 \%$ (standard error [SE] 5.1\%) after 1 year, 61.6\% (SE 7.4\%) after 2 years, $58.4 \%$ (SE7.7\%) after 3 years, and $47.8 \%$ (SE 9.2\%) after 4 years. The median OS could not be calculated, as $30.7 \%$ of the patients died and the median OS can only be calculated when half the patients die.

Throughout all five re-treatment cycles in 83 patients (Online Resource 4) with totally 141 re-treatment cycles (Online Resource 5), 6 patients had tumor progression according to the mRECIST criteria (after first cycle: $n=2$; after second cycle: $n=1$; third cycle: $n=2$; fourth cycle: $n=1$; fifth cycle: $n=0$ ). Radiological progression was detected in six patients (4.26\%), with a median PFS of 2.23 months (SE 2.43 months) (Fig. 2). Two of these patients died at the end of the follow-up period, which resulted in a median TTP of 7.81 months (SE 2.49 months) (Fig. 3).

Although the exclusion and inclusion criteria (Tab.1) were strictly endorsed, five patients in our cohort had advanced stages of HCC. They were relatively young at the time of diagnosis (58-64 years), and none of these patients developed PD during the DEB-TACE therapy. All tumors responded to DEB-TACE, and three patients even reached CR status. Four of the five patients with advanced liver disease died. The time range between the last TACE treatment and the date of death was $113.0 \pm 93.1$ days. The death was caused by decompensated cirrhotic liver disease and its consequences.

\section{Safety}

AEs were reported in 25 cases, documented in Online Resource 6 . A groin hematoma $(0.7 \%)$ and a hypertensive crisis $(0.7 \%)$, which occurred during the procedure, were found to be unrelated to the use of $40-\mu \mathrm{m}$ microspheres. Two female patients developed acute pancreatitis after 
Fig. 1 Overall survival

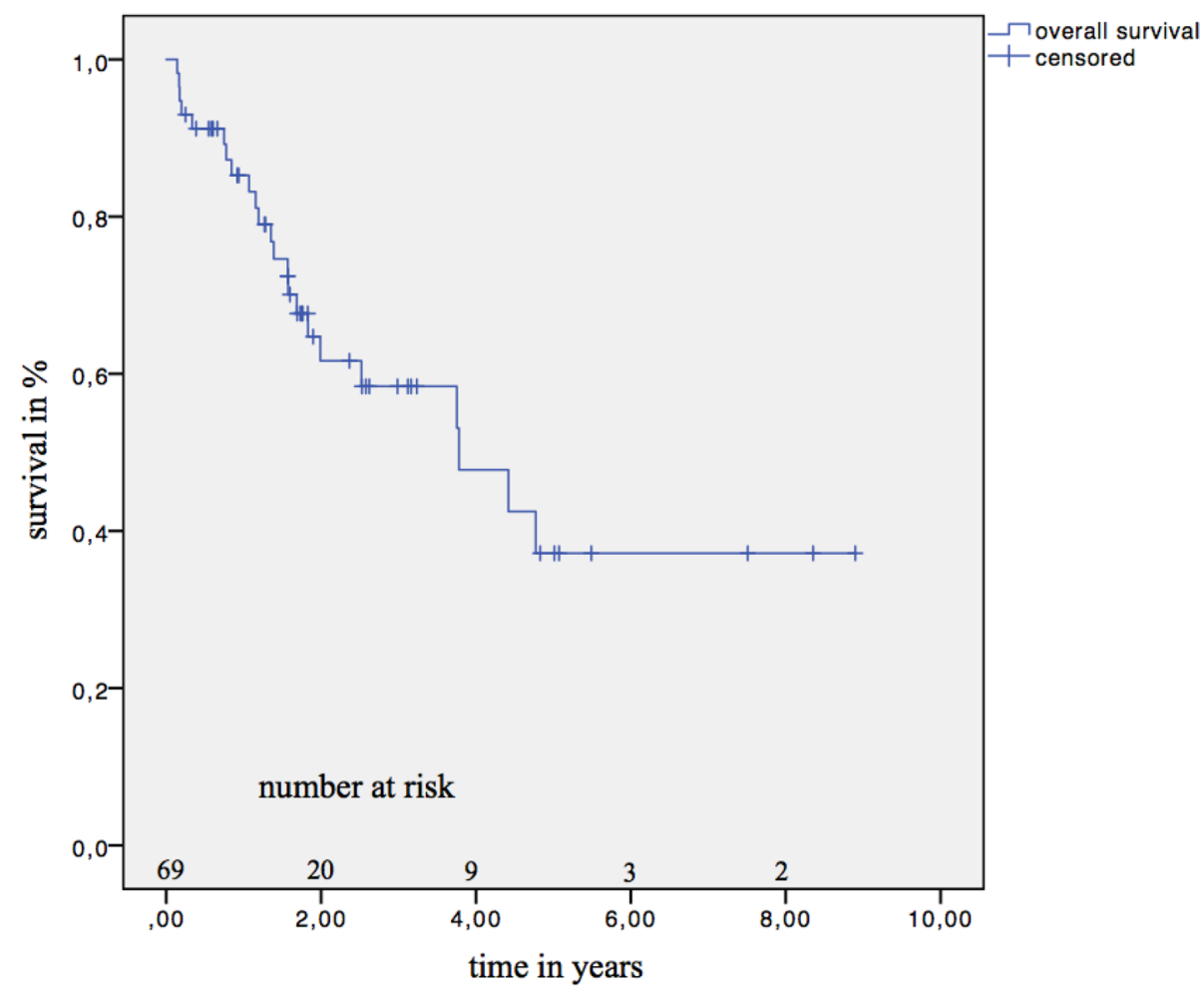

Fig. 2 Progression-free survival

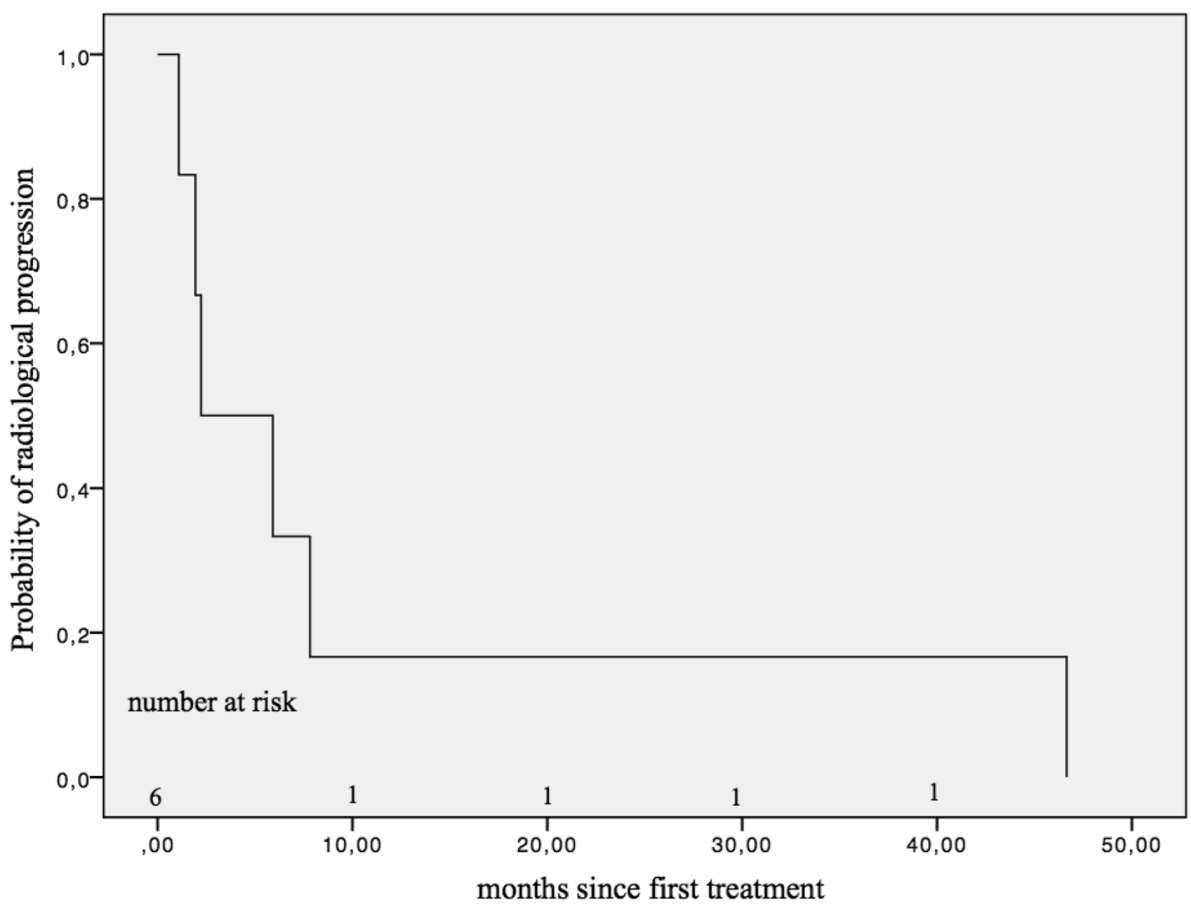

DEB-TACE treatment. In both cases, prolongation of the hospital stay was necessary. It remains unclear whether the pancreatitis was caused by the use of the $40 \mu \mathrm{m}$-sized microspheres. PES with prolongation of the hospital stay was observed in 12 cases (Online Resource 7). These patients had a large tumor burden and received, therefore, a high amount of embolic agent thus resolving in a high doxorubicin amount.

One SAE was recorded due to death from acute liver failure 16 days after the DEB-TACE intervention. The 
Fig. 3 Time to progression

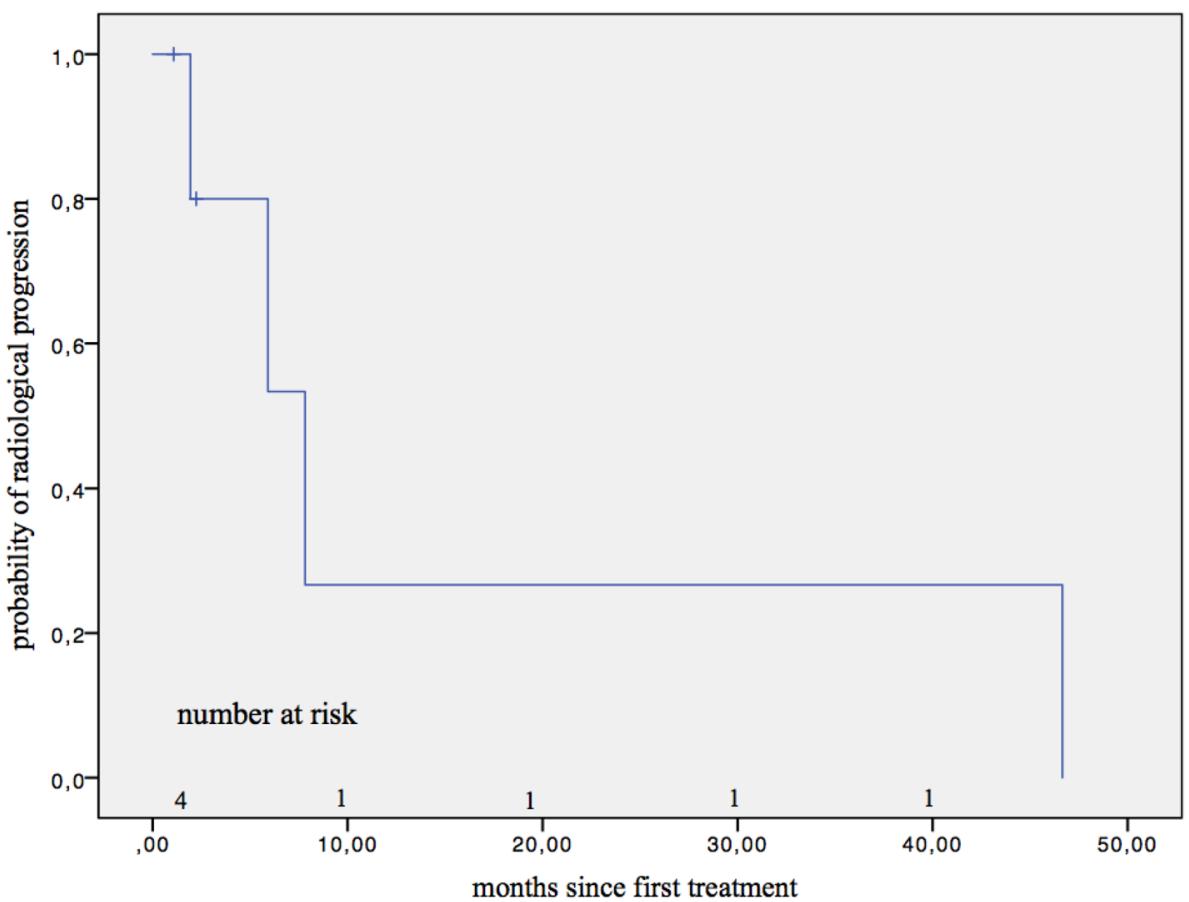

longitudinal axis of the tumor decreased from the baseline to the follow-up CT examination by $>56 \%$ (from 138.60 to $61.50 \mathrm{~mm}$ ). The high tumor reduction led to tumor lysis syndrome with respiratory, kidney, and, finally, liver failure. The SAE rate was $10.64 \%$, considering the one case of death, the 12 cases of PES, and the two cases of acute pancreatitis.

\section{Radiological tumor response}

The therapy response rate was determined with two different methods. First, a "target response analysis" was performed considering only the treated lesions, and second, an analysis that included all HCC lesions. The results differed in 18 cases as shown in Table 3.

The response rate according to the mRECIST criteria for the target response analysis (Table 3 ) was in most cases considered as either CR $(39.72 \%, n=56 / 141)$ or SD $(32.62 \%$, $n=46 / 141)$. The response rate $(\mathrm{CR}+\mathrm{PR})$ was $63.12 \%$. The disease control rate $(\mathrm{CR}+\mathrm{PR}+\mathrm{SD})$ was $95.74 \%$. The analysis also included an evaluation of whether ascites and/or portal venous thrombosis was present at each investigational time point as shown in Online Resource 8. In this study, the incidence of ascites increased between the pretreatment and post-treatment imaging for the first and second DEB-TACE cycles. The incidence of portal venous thrombosis remained the same in the pre- and post-treatment scans. In three cases, portal vein thrombosis was due to tumoral infiltration.

The correlation between the longitudinal axis and volume was examined. The $\mathrm{r}$ value was 0.954 , which indicated a significant correlation between the longitudinal axis and volume ( $\mathrm{p}<0.001)$ (Fig. 4).

The reduction of volume $(46.1 \pm 181.8$ to $28.0 \pm 146.3 \mathrm{ml})$, longitudinal axis $(36.8 \pm 28.21$ to $19.6 \pm 30.9 \mathrm{~mm})$, and short axis $(23.2 \pm 17.1$ to $11.0 \pm 17.2 \mathrm{~mm})$ in the treated lesions before and after each treatment was statistically significant, at $p=0.0001$.

The histogram analysis showed alterations in entropy, uniformity, MPP, UPP, and minimal and maximal density (all, $p<0.03$ ). For kurtosis, middle density, standard deviation, and skewness, the data before and after each treatment cycle did not change significantly.

\section{Discussion}

In this retrospective analysis, the main focus was to examine the safety and therapy response rate of $40-\mu \mathrm{m}$ microspheres for the treatment of HCC by investigating the tumor characteristics, such as the longitudinal axis, volume, and the parameters included in the histogram analysis after the treatment.

The therapy response rate was estimated by the use of the medical software mint lesion ${ }^{\mathrm{TM}}$, which also included a histogram analysis. To our knowledge, this is the first investigation in patients with $\mathrm{HCC}$, that contained this kind of analysis. We determined that a correlation between longitudinal axis and volume existed and that a decrease in the longitudinal diameter also resulted in a decline of volume. 
Fig. 4 Correlation between volume and the longitudinal axis

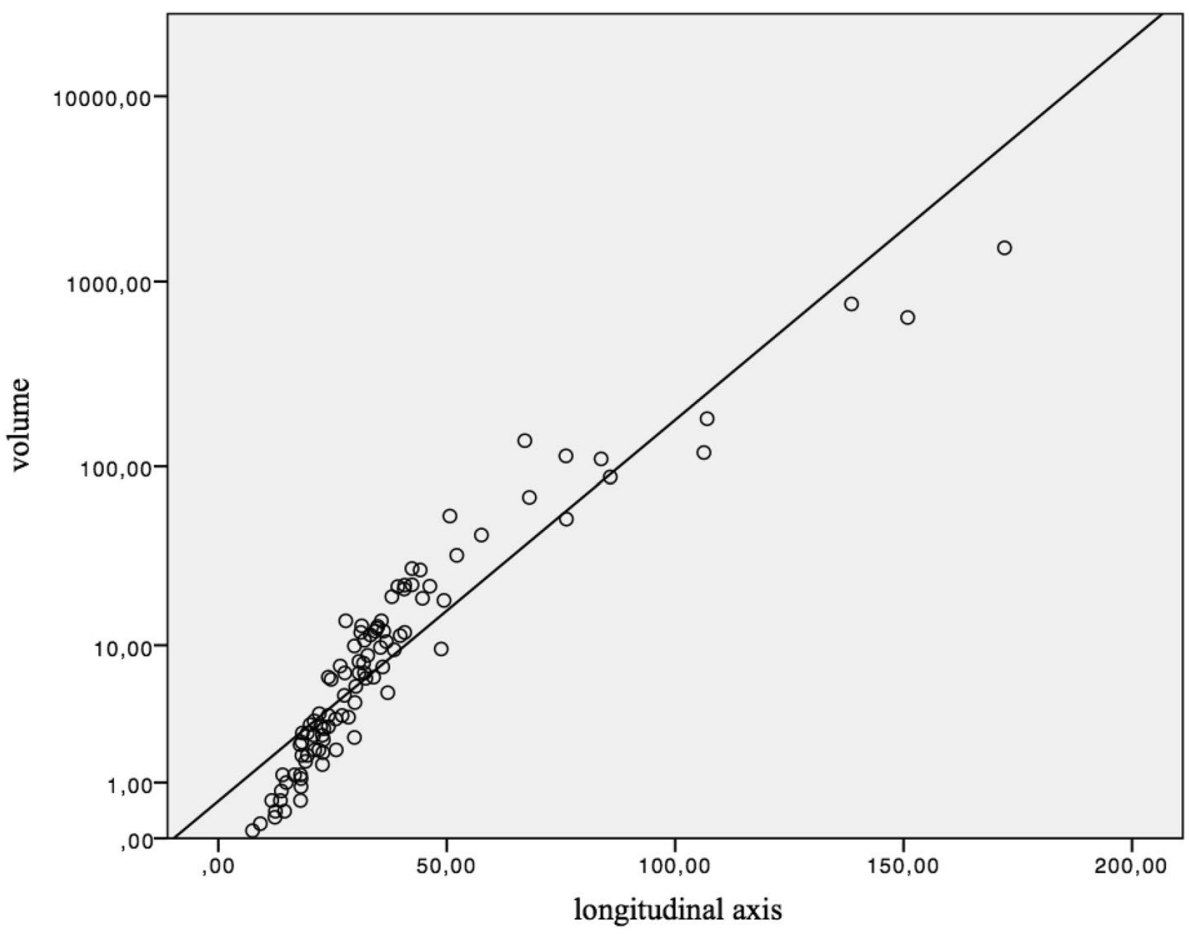

This analysis did not include a comparison group (e.g., different particle sizes or conventional TACE group); therefore, it cannot be claimed with certainty that the study outcomes are specific to the use of $40 \mu \mathrm{m}$-sized microspheres. Recently, Greco et al. demonstrated in a retrospective study the safety profile and response rate of $40-\mu \mathrm{m}$ doxorubicinloaded microspheres. Their study population consisted of 48 patients with early intermediate $\mathrm{HCC}$, who showed an objective response rate of $72.6 \%$ according to mRECIST (Greco et al. 2017). In comparison, this study population was larger, with 83 patients, but achieved comparable therapeutic success, with an objective therapy response rate of $63.12 \%$.

In 2011, Huppert et al. stated that the grade and duration of PES symptoms depend on the size of the tumor and surrounding liver tissue affected by TACE and on the amount of embolic material applied (Huppert 2011). In the animal study of Dinca et al. in which small microspheres of 30-60 $\mu \mathrm{m}$ provided a very effective distal occlusion and distribution of microspheres in the embolized territory than microsphere of 50-100 $\mu \mathrm{m}$. (Dinca et al. 2012) The usage of tightly calibrated microspheres in the size of $40 \mu \mathrm{m}$ also allows a deeper penetration of the nourishing tumor vessel and substantially accumulates the chemotherapeutic agent doxorubicin only in the tumor itself. Malagari et al. declared that distal embolization is desirable to avoid hypoxiainduced neoangiogenesis. (Malagari et al. 2014)

Our study findings support this claim, as the 12 patients who developed PES were the ones who received a high dose of doxorubicin $(95.5 \pm 50.8 \mathrm{mg})$ and consequently also a high amount of embolic agent. This group of patients had large HCC nodules, but they were also the ones with a good therapy response rate $(\mathrm{CR}=5 / 12, \mathrm{PR}=3 / 12, \mathrm{SD}=4 / 12)$. Prajapati et al. demonstrate that with different sizes of microspheres larger areas of necrosis may be due to the increased surface area of smaller beads inducing a greater burst release of doxorubicin within the tumor (Prajapati et al. 2014).

The SAE rate in this analysis was $10.64 \%$, including the 12 patients who developed PES, 1 case of death 16 days after the DEB-TACE cycle, and 2 cases of acute pancreatitis. Table E6 lists the symptoms that were monitored after 141 DEB-TACE interventions. In our patient cohort, only two patients developed a liver abscess with increased liver and infection parameters.

Dhamija et al. stated that biliary complications are uncommon $(0.87 \%)$ and that the biliary ducts have an exclusive blood supply from the hepatic artery branches; as a consequence of hepatic artery embolization, it is possible that an inadvertent occlusion may occur during TACE (Dhamija et al. 2015). None of these biliary complications were monitored in this study. However, two cases of acute pancreatitis after DEB-TACE were observed. The pancreatitis could have been caused by reflux of loaded microspheres due to potential communication channels around the common bill duct and liver hilum between the hepatic and pancreaticoduodenal arteries.

Interestingly, a prospective trial by Lee et al. found that 100-300- $\mu \mathrm{m}$ microspheres (DC Bead; Biocompatible, Farnham, UK) had excellent efficacy, but a relatively high overall incidence of PES at $67.6 \%$ in that cohort. Furthermore, in $19.7 \%$ of the patients, imaging showed prominent biliary 
injury (Lee et al. 2015). In comparison, in this study, the PES rate was fairly low, which could not only be related to the study's retrospective nature, but could also indicate a tendency of lower complications due to the application of smaller sized microspheres.

The limitations of this study included its retrospective character and the monocentric implementation that led to an unavoidable selection bias of patients. Moreover, no control group was enrolled. Conventional TACE (c-TACE) with lipiodol emulsion is still considered the gold standard; therefore, a comparison between 40- $\mu \mathrm{m}$ DEB-TACE and c-TACE is recommended to accumulate additional clinical evidence.

\section{Conclusion}

In conclusion, this retrospective analysis supports the hypothesis that a high response rate in the treatment of HCC can be achieved using small-sized microspheres, which allow a deeper penetration of the nourishing tumor vessel. This technique also minimizes the undesirable side effects of a false embolization.

Acknowledgements The authors wish to thank the research coordinator Laura Graziani and study assistant Elisabeth Lammers for the manuscript editing and the RECIST evaluation at the radiology research office, University Hospital Jena.

Author contributions KA and UT contributed to the conception, design and interpretation of the data analysis and were responsible for the drafting of the manuscript. KA, UT, RA, ID and NK revised the manuscript and gave final approval for submission.

Funding The study was supported by a research grant of Boston Scientific. Open Access funding provided by Projekt DEAL.

Availability of data material The data that support the findings of this study will be made available after sponsor approval (Friedrich-SchillerUniversity Jena, Germany). In particular, individual participant data that underlie the results reported in this article (text, tables, figures, appendices), and other patient data will be available strictly for scientific reasons and only on demand. Source data will be made available especially for systematic reviews and meta-analysis.

Code availability The software application used for the image evaluation of therapeutic response was Mint Lesion ${ }^{\mathrm{TM}}$ (Mint Medical, Dossenheim, Germany).

\section{Compliance with ethical standards}

Competing interest All other authors declare that they have no conflict of interest, except of Prof. Teichgräber who received a funding for the retrospective study by Boston Scientific.

Ethical approval The retrospective data analysis has been approved by the appropriate institutional ethics committee of the University Hospital Jena with the protocol number 5294-10/17 on October 24th 2017 and has been performed in accordance with the ethical standards as laid down in the 1964 Declaration of Helsinki and its later amendments or comparable ethical standards.

Consent to participate Informed consent of the evaluated subjects is not required for a retrospective data analysis.

Consent for publication All authors have read and approved the manuscript for publication.

Open Access This article is licensed under a Creative Commons Attribution 4.0 International License, which permits use, sharing, adaptation, distribution and reproduction in any medium or format, as long as you give appropriate credit to the original author(s) and the source, provide a link to the Creative Commons licence, and indicate if changes were made. The images or other third party material in this article are included in the article's Creative Commons licence, unless indicated otherwise in a credit line to the material. If material is not included in the article's Creative Commons licence and your intended use is not permitted by statutory regulation or exceeds the permitted use, you will need to obtain permission directly from the copyright holder. To view a copy of this licence, visit http://creativecommons.org/licenses/by/4.0/.

\section{References}

Abbasi A, Bhutto AR, Butt N, Munir SM (2012) Corelation of serum alpha fetoprotein and tumor size in hepatocellular carcinoma. JPMA 62:33

Dhamija E, Paul SB, Gamanagatti SR, Acharya SK (2015) Biliary complications of arterial chemoembolization of hepatocellular carcinoma. Diagn Interv Imaging 96:1169-1175. https://doi. org/10.1016/j.diii.2015.06.017

Dhanasekaran R, Kooby DA, Staley CA, Kauh JS, Khanna V, Kim HS (2010) Comparison of conventional transarterial chemoembolization (TACE) and chemoembolization with doxorubicin drug eluting beads (DEB) for unresectable hepatocelluar carcinoma (HCC). J Surg Oncol 101:476-480. https://doi.org/10.1002/jso.21522

Greco G, Cascella T, Facciorusso A et al (2017) Transarterial chemoembolization using 40 microm drug eluting beads for hepatocellular carcinoma. World J Radiol 9:245-252. https://doi. org/10.4329/wjr.v9.i5.245

Han S, Zhang X, Zou L et al (2014) Does drug-eluting bead transcatheter arterial chemoembolization improve the management of patients with hepatocellular carcinoma? A meta-analysis. PLoS ONE 9:e102686. https://doi.org/10.1371/journal.pone.0102686

Huppert P (2011) Current concepts in transarterial chemoembolization of hepatocellular carcinoma. Abdom Imaging 36:677-683. https ://doi.org/10.1007/s00261-011-9755-4

Lammer J, Malagari K, Vogl T, Pilleul F, Denys A, Watkinson A, Pitton M, Sergent G, Pfammatter T, Terraz S, Benhamou Y (2010) Prospective randomized study of doxorubicin-eluting-bead embolization in the treatment of hepatocellular carcinoma: results of the precision v study. Cardiovasc Intervent Radiol 33(1):41-52

Lee S, Kim BK, Kim SU et al (2015) Early alpha-fetoprotein response predicts survival in patients with advanced hepatocellular carcinoma treated with sorafenib. J Hepatocell Carcinoma 2:39-47. https://doi.org/10.2147/jhc.s79353

Lencioni R, Llovet JM (2010) Modified RECIST (mRECIST) assessment for hepatocellular carcinoma. Semin Liver Dis 30:52-60. https://doi.org/10.1055/s-0030-1247132

Lencioni R, Kudo M, Ye SL et al (2014) GIDEON (Global Investigation of therapeutic DEcisions in hepatocellular carcinoma and of 
its treatment with sorafeNib): second interim analysis. Int J Clin Pract 68:609-617. https://doi.org/10.1111/ijcp.12352

Lencioni R, de Baere T, Soulen MC, Rilling WS, Geschwind JF (2016) Lipiodol transarterial chemoembolization for hepatocellular carcinoma: a systematic review of efficacy and safety data. Hepatology 64:106-116. https://doi.org/10.1002/hep.28453

Malagari K, Pomoni M, Moschouris H et al (2014) Chemoembolization of hepatocellular carcinoma with HepaSphere 30-60 mum Safety and efficacy study. Cardiovasc Intervent Radiol 37:165-175. https ://doi.org/10.1007/s00270-013-0777-x

Mazzaferro V, Llovet JM, Miceli R, Bhoori S, Schiavo M, Mariani L, Camerini T, Roayaie S, Schwartz ME, Grazi GL, Adam R, Neuhaus P, Salizzoni M, Bruix J, Forner A, De Carlis L, Cillo U, Burroughs AK, Troisi R, Rossi M, Gerunda GE, Lerut J, Belghiti J, Boin I, Gugenheim J, Rochling F, Van Hoek B, Majno P, on behalf of the Metroticket Investigator Study Group (2009) Predicting survival after liver transplantation in patients with hepatocellular carcinoma beyond the Milan Criteria: a retrospective, exploratory analysis. Lancet Oncol 10:35-43. https://doi.org/10.1016/s1470-

Moore TJ, Cohen MR, Furberg CD (2007) Serious adverse drug events reported to the Food and Drug Administration. Arch Int Med 167(16):1752-1759

Nakamura TTA, Yamamoto S, Fukuda H (2012) Evaluation of three definitions of progression-free survival in preoperative cancer therapy. Jpn J Clin Oncol 42:896-902

Nam HC, Jang B, Song MJ (2016) Transarterial chemoembolization with drug-eluting beads in hepatocellular carcinoma. World $\mathrm{J}$
Gastroenterol 22:8853-8861. https://doi.org/10.3748/wjg.v22. i40.8853

Prajapati HJ, Xing M, Spivey JR et al (2014) Survival, efficacy, and safety of small versus large doxorubicin drug-eluting beads TACE chemoembolization in patients with unresectable HCC. AJR Am J Roentgenol 203:W706-W714. https://doi.org/10.2214/ ajr.13.12308

Saad EDKA (2008) Progression-free survival and time to progression as primary end points in advanced breast cancer: often used, sometimes loosely defined. Ann Oncol 20:460-464

Sattler T, Bredt C, Surwald S, Rust C, Rieger J, Jakobs T (2018) Efficacy and safety of drug eluting bead RACE with microspheres < $150 \mu \mathrm{m}$ for the treatment of hepatocellular carcinoma. Anticancer Res 38:1025-1032

Dinca H PJ, Baylatry MT, Ghegediban SH, Pascale F, Manfait M et al. Why do small size doxorubicin-eluting micropsheres induce more tissue necrosis than larger ones? A comparative study in healthy pig liver. Oral Commun 2012; 2206

Publisher's Note Springer Nature remains neutral with regard to jurisdictional claims in published maps and institutional affiliations. 